Roy A. Mimna, 57 West Liberty Street, Hubbard, Ohio 44425,

e-mail:mimna@aol.com

Eric J. Wingler, Department of Mathematics and Statistics, Youngstown

State University, Youngstown, Ohio 44555, e-mail:wingler@math.ysu.edu

\title{
LOCALLY BOUNDED FUNCTIONS
}

\section{Introduction}

A well known property of continuous real-valued functions $f: X \rightarrow \mathbf{R}$, where $X$ is a topological space, is that for any $x \in X, f$ is bounded on some neighborhood of $x$. Here we discuss this property of continuous functions, which we shall call "local boundedness", in a more general context. R. V. Fuller's "subcontinuity" is shown to be equivalent to local boundedness. The classical theorem that a continuous real-valued function on a compact space is bounded, is generalized and shown to be true for the larger class of locally bounded functions. Certain classes of discontinuous functions are shown to be locally bounded, and properties of locally bounded functions are studied.

Throughout this paper, $X$ and $Y$ denote topological spaces, with no special properties unless otherwise indicated.

\section{Basic Definitions}

Definition 1. A function $f: X \rightarrow M$, where $M$ is a metric space, is locally bounded if for any $x$ in $X$ there exists an open set $U$ containing $x$ such that $f$ is bounded on $U$.

If $f: X \rightarrow M$ is a continuous function and $x$ is any point in $X$, then $f$ is bounded on the open set $f^{-1}[B(f(x), 1)]$, where $B(f(x), 1)$ denotes an open ball in $M$ of radius 1 . Hence we have the following theorem.

Theorem 1. If $M$ is a metric space and $f: X \rightarrow M$ is a continuous function, then $f$ is locally bounded.

Key Words: locally bounded functions, locally compact functions, subcontinuity

Mathematical Reviews subject classification: 26A15

Received by the editors November 1, 1996 
In [1], J. Doboš applied the term "locally bounded" to functions in a manner different from the present usage. In this paper these functions shall be called "locally compact" in order to distinguish between the two concepts.

Definition 2. A function $f: X \rightarrow Y$ is locally compact at $x$ in $X$ if there exists a compact subset $K$ of $Y$ such that $x \in \operatorname{Int}\left[f^{-1}(K)\right]$.

It is easy to see that the concepts, "locally bounded" and "locally compact", are not equivalent; for if $i$ is the identity mapping from the set of reals with the usual metric into the the set of reals with the discrete metric, then $i$ is locally bounded but not locally compact. However, if a function is locally compact and its codomain is a metric space, then it is also locally bounded.

The term "locally compact" is more general than "locally bounded" since it can be applied to functions whose codomains are topological spaces. A locally bounded function must have a metric space as its codomain. Also, it is possible for a function $f: X \rightarrow M$ to be locally bounded under one metric on $M$ and not locally bounded under an equivalent metric on $M$. For example, consider the usual metric on $\mathbf{R}$ and the metric $\rho$ defined by $\rho(x, y)=|x-y| /(1+|x-y|)$. These are equivalent metrics on $\mathbf{R}$, but since the latter is bounded, any function from $X$ into $(\mathbf{R}, \rho)$ is locally bounded.

\section{A Condition Equivalent to Local Boundedness}

The notion of subcontinuity was introduced in [2] and has been studied in [5].

Definition 3. A function $f: X \rightarrow Y$ is subcontinuous if for any net $\left(x_{a}\right)$ in $X$ such that $\left(x_{a}\right)$ converges to $p$ in $X$, there is a subnet, $\left(f\left(x_{s(b)}\right)\right)$, of $\left(f\left(x_{a}\right)\right)$ that converges to some point $q$ in $Y$.

Theorem 2. Let $f: X \rightarrow M$ be a function, where $X$ is a first countable Hausdorff space and $M$ is a metric space. A sufficient condition that $f$ be locally bounded is that $f$ be subcontinuous. If $M=\mathbf{R}$, then the condition is also necessary.

Proof. We will prove sufficiency first. Assume $f$ is not locally bounded at some point $x_{0}$ in $X$, and let $d$ be the metric on $M$. Let

$$
D=\left\{(U, n): U \text { is a neighborhood of } x_{0} \text { and } n \in \mathbf{N}\right\} .
$$

Then $D$ can be directed by the relation $\leq$, where $(U, n) \leq(V, m)$ if and only if $V \subset U$ and $n \leq m$. Since $f$ is not locally bounded at $x_{0}$, for each $a=(U, n)$ in $D$ there is an element $x_{a}$ in $U$ such that $d\left(f\left(x_{a}\right), f\left(x_{0}\right)\right)>n$. As can be 
easily shown, the net $\left(x_{a}: a \in D\right)$ converges to $x_{0}$. Since $f$ is subcontinuous, there exists a subnet $\left(x_{s(a)}: a \in D^{\prime}\right)$ such that $\left(f\left(x_{s(a)}\right)\right)$ converges to some point $y$ in $M$. Let $m$ be a natural number such that $d\left(f\left(x_{0}\right), y\right) \leq m$. There exists $a \in D^{\prime}$ such that $d\left(f\left(x_{s(b)}\right), y\right)<1$ for all $b \in D^{\prime}$ such that $b \geq a$. Hence

$$
d\left(f\left(x_{0}\right), f\left(x_{s(b)}\right)\right)<m+1
$$

for all $b \in D^{\prime}$ such that $b \geq a$. There exist a natural number $k>m+1$ and a neighborhood $U$ of $x_{0}$ such that $(U, k) \geq s(a)$. Also, there exists $b \in D^{\prime}$ with $b \geq a$ such that $s(b) \geq(U, k)$. This implies that $d\left(f\left(x_{0}\right), f\left(x_{s(b)}\right)\right)>m+1$, which contradicts $(*)$. Hence $f$ is locally bounded.

Now suppose that $f: X \rightarrow \mathbf{R}$ is locally bounded. Let $\left(x_{a}\right)$ be any net in $X$ converging to a point $p$. Since $f$ is locally bounded, there exist an open set $U$ containing $p$ and a number $M$ such that $|f(x)| \leq M$ for all $x \in U$. Since $\left(x_{a}\right)$ eventually lies in $U,\left(f\left(x_{a}\right)\right)$ eventually lies in $[M,-M]$, which is compact. Hence there is a subnet $\left(x_{s(a)}\right)$ such that $\left(f\left(x_{s(a)}\right)\right)$ converges to some point in $[M,-M]$.

Remark 1. The necessity part of this theorem remains valid if $\mathbf{R}$ is replaced by a metric space whose closed balls are compact.

A comparable equivalence between subcontinuity and local compactness can also be shown. The necessity portion of the proof of the next theorem requires the following theorem of Fuller [2, Theorem 2.1]: If $f: X \rightarrow Y$ is a subcontinuous function, where $Y$ is completely regular, then for each compact subset $K$ of $X$, the set $\overline{f(K)}$ is compact.

Theorem 3. Let $f: X \rightarrow Y$ be a function, where $Y$ is Hausdorff. A sufficient condition that $f$ be subcontinuous is that $f$ be locally compact. If $X$ is locally compact and $Y$ is completely regular, the condition is also necessary.

Proof. To prove sufficiency, let $\left(x_{a}\right)$ be a net in $X$ converging to a point $x$ in $X$. Since $f$ is locally compact, there is a compact subset $K$ of $Y$ such that $x \in \operatorname{Int}\left[f^{-1}(K)\right]$. Since $\left[f^{-1}(K)\right]$ contains the tail of $\left(x_{a}\right)$, the net $\left(f\left(x_{a}\right)\right)$ is eventually in $K$; hence $\left(f\left(x_{a}\right)\right)$ contains a convergent subnet.

To show necessity, suppose $f: X \rightarrow Y$ is a subcontinuous function, where $X$ is locally compact and $Y$ is completely regular. Let $x$ be any point in $X$. Then there is a compact neighborhood $K$ of $x$. By Theorem 2.1 of [2], the set $M=\overline{f(K)}$ is compact, and $x \in \operatorname{Int}\left[f^{-1}(M)\right]$.

Remark 2. Since continuous functions are subcontinuous, it is immediate that continuous functions from locally compact spaces into completely regular spaces are locally compact. 


\section{Functions which are Locally Bounded}

As we have seen, subcontinuous functions are locally bounded. The question naturally arises as to what other kinds of noncontinuous functions are locally bounded.

Evidently, if a real-valued function $f: \mathbf{R} \rightarrow \mathbf{R}$ has a removable discontinuity at a point $x_{0}$, then it is bounded on a neighborhood of $x_{0}$, for since $f$ approaches a limit $L$ at $x_{0}$, there is a neighborhood $U$ of $x_{0}$ such that $|f(x)-L|<1$ for all $x$ in $U-\left\{x_{0}\right\}$. Thus, $f$ is bounded on $U$ by $\max \left\{|L|+1,\left|f\left(x_{0}\right)\right|\right\}$.

From [3] and [4] we have the following:

Definition 4. A function $f: X \rightarrow Y$ has at worst a removable discontinuity at a point $x$ of $X$ if there is a point $y$ in $Y$ such that for each open set $V$ containing $y$, there is an open set $U$ containing $x$ such that $f(U-\{x\}) \subset V$.

Theorem 4. Let $f: X \rightarrow M$ be a function, where $M$ is a metric space. If $f$ has at worst a removable discontinuity at a point $x_{0}$ in $X$, then $f$ is bounded on some open set containing $x_{0}$; that is, $f$ is locally bounded at $x_{0}$.

Proof. If $f$ has at worst a removable discontinuity at the point $x_{0}$, then there exists an open ball $B(y, 1)$ in $M$ and an open set $U$ containing $x_{0}$ such that $f\left(U-\left\{x_{0}\right\}\right) \subset B(y, 1)$. Since $B(y, 1)$ is a bounded subset of $M$, the set $B(y, 1) \cup\left\{f\left(x_{0}\right)\right\}$ is also a bounded subset of $M$. Hence $f$ is locally bounded at $x_{0}$.

Upper or lower semicontinuity is not a sufficient condition for a function to be locally bounded. Let

$$
f(x)= \begin{cases}\ln |x|, & \text { if } x \neq 0 \\ 0, & \text { if } x=0\end{cases}
$$

Then $f$ is upper semicontinuous, but it is not locally bounded at 0 .

Since a bounded function is also locally bounded, it is easy to find examples of nonmeasurable locally bounded functions; for example, consider the characteristic function $\chi_{P}$, where $P$ is a nonmeasurable set.

Consider the function $f:(0,1) \rightarrow \mathbf{R}$ that is 0 at each irrational number and has the value $q$ at each rational number $\frac{p}{q}$, where $p$ and $q$ are relatively prime natural numbers. This function is not locally bounded at any point of $(0,1)$. 


\section{Properties of Locally Bounded Functions}

It is evident that, since a continuous function is not necessarily bounded on its domain, a locally bounded function need not be bounded on its domain. However, if the domain of a continuous function is compact, then the function is bounded. The same thing is true of locally bounded functions.

Theorem 5. Let $X$ be a compact space, and let $f: X \rightarrow \mathbf{R}$ be a locally bounded function. Then $f$ is bounded.

Proof. The proof of this is essentially the same as that used for continuous functions on compact spaces. The space $X$ can be covered with open sets on which $f$ is bounded. Since $X$ is compact, it can be covered with a finite number of these open sets. Hence, $f$ is bounded on $X$.

In the preceding theorem, it is not enough that $X$ be locally compact as the example of the identity function $i: \mathbf{R} \rightarrow \mathbf{R}$ clearly illustrates.

Theorem 6. If $f: X \rightarrow \mathbf{R}$ is locally bounded and has a closed graph, then $f$ is continuous.

Proof. (Corollary of Theorem 3.4 [2]).

Corollary 1. Suppose that the function $f: \mathbf{R} \times \mathbf{R} \rightarrow \mathbf{R}$ has a closed graph, and that for each $x$ the functions $f_{x}$ and $f^{x}$, defined by $f_{x}(y)=f(x, y)$ and $f^{x}(y)=f(y, x)$, are locally bounded. Then $f$ is continuous.

Proof. By Theorem 6 the function $f$ is separately continuous. Thus $f$ is continuous by Theorem 1 of [6].

\section{Spaces of Locally Bounded Functions}

In this section we will examine certain spaces of locally bounded functions. Let $\mathcal{L B}(X, M)$ denote the set of all locally bounded functions from $X$ into $M$, where $M$ is a metric space. A natural topology to consider for $\mathcal{L B}(X, M)$ is the compact-open topology, which has as a subbasis sets of the form

$$
(K, U)=\{f \in \mathcal{L} \mathcal{B}(X, M): f(K) \subset U\},
$$

where $K$ is compact and $U$ is open.

It is easy to show that $\mathcal{L B}(X, M)$ with the compact-open topology is Hausdorff; however, we will show a slightly stronger result.

Theorem 7. Let $x \in X$ and let $\omega_{x}: \mathcal{L B}(X, M) \rightarrow M$ be the point evaluation map defined by $\omega_{x}(f)=f(x)$. Then $\omega_{x}$ is continuous. 
Proof. Let $f \in \mathcal{L B}(X, M)$ and let $U$ be a neighborhood of $f(x)$. If $g \in$ $(\{x\}, U)$, then $\omega_{x}(g)=g(x) \in U$. Hence $\omega_{x}$ is continuous.

Theorem 8. The space $\mathcal{L B}(X, M)$ with the compact-open topology is $T_{2 \frac{1}{2}}$, that is, each pair of points can be separated with closed neighborhoods of the points.

Proof. Let $f$ and $g$ be distinct points in $\mathcal{L B}(X, M)$. Then there exists $x \in X$ such that $f(x) \neq g(x)$. Since $M$ is a metric space, it is normal. Hence there exist disjoint neighborhoods $U_{f}$ and $U_{g}$ of $f(x)$ and $g(x)$, respectively, such that $\bar{U}_{f}$ and $\bar{U}_{g}$ are disjoint. Since $\omega_{x}$ is continuous, $\omega_{x}^{-1}\left(\bar{U}_{f}\right)$ and $\omega_{x}^{-1}\left(\bar{U}_{g}\right)$ are disjoint closed neighborhoods of $f$ and $g$.

The space $\mathcal{L B}(X, M)$ is not, in general, regular. Let $I$ denote the closed interval $[0,1]$. Then we have the following theorem.

Theorem 9. The space $\mathcal{L B}(I, \mathbf{R})$ with the compact-open topology is not regular.

Proof. Let

$$
f(x)= \begin{cases}x, & \text { if } x \in \operatorname{Int}(I), \\ \frac{1}{2}, & \text { otherwise }\end{cases}
$$

Then $f \in \mathcal{L B}(I, \mathbf{R})$ and $f(I)=\operatorname{Int}(I)$. Hence $f \in(I$, $\operatorname{Int}(I))$. Suppose that $\mathcal{L B}(I, \mathbf{R})$ is regular. Then there exist compact sets $K_{1}, \ldots, K_{n}$ and open sets $U_{1}, \ldots, U_{n}$ such that

$$
f \in \bigcap_{i=1}^{n}\left(K_{i}, U_{i}\right) \subset \overline{\bigcap_{i=1}^{n}\left(K_{i}, U_{i}\right)} \subset(I, \operatorname{Int}(I)) .
$$

(Without any loss of generality it may be assumed that $U_{i} \subset I$ for each $i$.) This implies that $\cup_{i=1}^{n} K_{i} \supset I$ and $\cup_{i=1}^{n} U_{i} \subset I$. Hence at least one set $K_{j}$ contains an increasing sequence $\left(x_{n}\right)$ such that $\lim x_{n}=1$. This sequence can be chosen so that if $x_{n} \in K_{i}$ for some $n$ and $i$, then $x_{n} \in K_{i}$ for all $n$. Hence each set $K_{i}$ either contains $\left(x_{n}\right)$ or it does not contain any term of $\left(x_{n}\right)$.

Let

$$
g(x)= \begin{cases}1, & \text { if } x=x_{n} \text { for some } n \\ f(x), & \text { otherwise. }\end{cases}
$$

Then $g \notin(I, \operatorname{Int}(I))$. We will show that $g \in \overline{\cap_{i=1}^{n}\left(K_{i}, U_{i}\right)}$ and thereby contra$\operatorname{dict}\left({ }^{*}\right)$. Let $\cap_{i=1}^{m}\left(J_{i}, V_{i}\right)$ be a basic open neighborhood of $g$. If $\cup_{i=1}^{m} J_{i}$ contains no element of the sequence $\left(x_{n}\right)$, then $f=g$ on $\cup_{i=1}^{m} J_{i}$; hence $f \in \cap_{i=1}^{m}\left(J_{i}, V_{i}\right)$ and $\left(\cap_{i=1}^{n}\left(K_{i}, U_{i}\right)\right) \cap\left(\cap_{i=1}^{m}\left(J_{i}, V_{i}\right)\right) \neq \emptyset$. Now suppose that $x_{j} \in \cup_{i=1}^{m} J_{i}$ for 
some $j$. Let $H=\cap\left\{U_{i}:\left(x_{n}\right) \subset K_{i}\right\}$. Since $f\left(\left(x_{n}\right)\right) \subset f\left(K_{i}\right) \subset U_{i}$ for each $i$ such that $\left(x_{n}\right) \subset K_{i}$, it follows that $f\left(\left(x_{n}\right)\right) \subset H$, and since $f\left(x_{n}\right)=x_{n}$ and $x_{n} \rightarrow 1$, we may conclude that $1 \in \bar{H}$. Now $x_{j} \in J_{i}$ for some $i$, so $1=g\left(x_{j}\right) \in g\left(J_{i}\right) \subset V_{i}$. Hence, if $G_{j}=\cap\left\{V_{k}: x_{j} \in J_{k}\right\}$, then $G_{j}$ is a neighborhood of 1 for each $j$ such that $x_{j} \in \cup_{i=1}^{m} J_{i}$. Thus, $G_{j} \cap H \neq \emptyset$ for each such $j$. For each such $j$ let $y_{n} \in G_{n} \cap H$. Let

$$
h(x)= \begin{cases}y_{n}, & \text { if } x=x_{n} \text { for some } n \text { and } x_{n} \in \cup_{i=1}^{m} J_{i}, \\ f(x), & \text { otherwise }\end{cases}
$$

Then $h \in\left(\cap_{i=1}^{n}\left(K_{i}, U_{i}\right)\right) \cap\left(\cap_{i=1}^{m}\left(J_{i}, V_{i}\right)\right)$. Hence $\mathcal{L B}(I, \mathbf{R})$ is not regular.

A well-known theorem on function spaces states that if $X$ is homeomorphic to $Y$ and $U$ is homeomorphic to $V$, then $U^{X}$ and $V^{Y}$ with the compact-open topology are homeomorphic. A similar but weaker statement can be made about $\mathcal{L B}(X, M)$.

Theorem 10. Let $g: X \rightarrow Y$ be continuous and let $T_{g}: \mathcal{L B}(Y, M) \rightarrow$ $\mathcal{L B}(X, M)$ be defined by $T_{g}(f)=f \circ g$. Then $T_{g}$ is continuous.

Proof. Let $f \in \mathcal{L B}(Y, M)$ and let $(K, V)$ be a subbasic open neighborhood of $f \circ g$. Then $f \circ g(K) \subset V$, and since $g(K)$ is compact, it follows that $f \in$ $(g(K), V)$. Now if $h \in(g(K), V)$, then $h \circ g \in(K, V)$; that is $T_{g}(h) \in(K, V)$. Hence $(g(K), V) \subset T_{g}^{-1}(K, V)$.

Corollary 2. If $X$ is homeomorphic to $Y$, then $\mathcal{L B}(X, M)$ is homeomorphic to $\mathcal{L B}(Y, M)$.

Proof. Let $h: X \rightarrow Y$ be a homeomorphism. It is easily verified that $T_{h}$ is bijective and that $T_{h}^{-1}=T_{h^{-1}}$. It follows from the previous theorem that $T_{h}$ and $T_{h}^{-1}$ are both continuous.

Remark 3. It is not clear whether or not $\mathcal{L B}\left(X, M_{1}\right)$ and $\mathcal{L B}\left(X, M_{2}\right)$ are homeomorphic when $M_{1}$ and $M_{2}$ are. Certainly, the natural attempt at defining a homeomorphism fails. For example, let $M_{1}=(0,1)$ and $M_{2}=\mathbf{R}$ with the usual metric. If $h: M_{1} \rightarrow M_{2}$ is a homeomorphism, then a natural function $H$ on $\mathcal{L B}\left(X, M_{1}\right)$ is defined by $H(f)=h \circ f$. However this does not work! Every function $f: X \rightarrow M_{1}$ is in $\mathcal{L B}\left(X, M_{1}\right)$, but $h \circ f$ is not necessarily locally bounded; hence $H$ does not map $\mathcal{L B}\left(X, M_{1}\right)$ into $\mathcal{L B}\left(X, M_{2}\right)$. Thus, we leave this as an open question.

\section{References}

[1] J. Doboš, On the set of points of discontinuity for functions with closed graphs, Čas. pěst. mat., 110 (1985), 60-68. 
[2] R. V. Fuller, Relations among continuous and various noncontinuous functions, Pac. J. Math., 25(3) (1968), 495-509.

[3] E. Halfar, Conditions implying continuity of functions, Proc. Amer. Math. Soc., 11 (1960), 688-691.

[4] T. R. Hamlett, The at worst removable discontinuities of functions and cluster sets, Math Chronicle, 4 (1976), 94-100.

[5] T. Noiri, Sequentially subcontinuous functions, Accad. Naz. dei Lincei, $\mathbf{5 8}$ (1975), 370-373.

[6] Z. Piotrowski and E. Wingler, A note on continuity points of functions, Real Analysis Exchange, 16 (1990-91), 408-414. 